\title{
Pulmonary rehabilitation in long COVID: more than just natural
}

\section{recovery!?}

Copyright (CThe authors 2021

This version is distributed under the terms of the Creative Commons Attribution NonCommercial Licence 4.0. For commercial reproduction rights and permissions contact permissions@ersnet.org

Received: 7 July 2021 Accepted: 9 July 2021

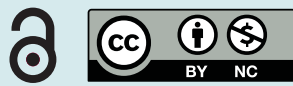

From the authors:

We thank A. Hussain and co-workers for their correspondence regarding our study on the benefits of pulmonary rehabilitation (PR) in coronavirus disease 2019 (COVID-19) [1].

Although a large proportion of COVID-19 patients recovers fully from the disease, approximately 5-10\% experience prolonged symptoms for several months following the acute COVID-19 phase [2-4]. This results in millions of people suffering from COVID-19 sequelae worldwide. Therefore, beneficial interventions are urgently needed to counteract these long-term consequences. A Cochrane review from April 2021 identified more than 50 studies that investigated rehabilitative interventions in post-COVID-19 patients [5]. Amongst them are two randomised controlled trials that included 72 and 140 post-acute patients, respectively, showing that respiratory techniques have superior benefits beyond natural recovery to improve pulmonary function, exercise performance, quality of life, and anxiety, especially in combination with group psychological interventions [6, 7]. However, we admit that there is currently no randomised controlled trial available that has investigated the effects of a more comprehensive, multidisciplinary PR programme in COVID-19.

Due to the lack of a control group in our study, the impact of natural recovery cannot be determined. Therefore, we agree with A. Hussain and co-workers that the natural convalescence after the acute COVID-19 phase may have contributed to the effects of PR in our study (as we discussed in the section about limitations). However, we would like to point out why we believe that PR seems to have additional benefits beyond spontaneous recovery.

A. Hussain and co-workers questioned why patients in the mild/moderate group without functional limitation were referred to PR "only" due to ongoing symptoms. The German COVID-19 rehabilitation guideline recommends referring patients to rehabilitation programmes when symptoms or other impairments of activities of daily living are ongoing [8]. A. Hussain and co-workers also mentioned a 6-min walk distance (6MWD) of 500-580 m to be "normal". Patients in our study reached a median 6MWD of $509 \mathrm{~m}$, which corresponded to only $71 \%$ predicted (according to the reference equation by Troosters et al. [9]). We agree that a higher baseline 6MWD leads to a certain ceiling effect that limits the chances to improve 6MWD after PR. Nevertheless, COVID-19 patients in the mild/moderate group were able to improve 6MWD by $48 \mathrm{~m}$ (interquartile range 35-113 m). This range is clearly beyond the expected minimal important difference of $30 \mathrm{~m}$ [10] and well beyond common variabilities seen in 6MWD [11]. Furthermore, since the severe acute respiratory syndrome coronavirus 2 (SARS-CoV-2) infection in the mild/moderate patient group had occurred 6 months before, the improvement in 6MWD during a comprehensive 3-week PR might be rather related to the intervention than to a spontaneous recovery after such a long period. Also, forced vital capacity (FVC) did not influence 6MWD in a relevant fashion: the correlations between the improvements in FVC and 6MWD were low and not significant (mild/moderate: $\mathrm{r}=-0.03$; severe/critical: $\mathrm{r}=0.33$ ).

A study by HuAng et al. [12] has shown that hospitalised COVID-19 patients had a median 6MWD of $495 \mathrm{~m}, 6$ months after hospital discharge. Patients in the severe/critical COVID-19 group in our study reached a comparable 6MWD (468 m) already at PR discharge (which was only 6 weeks after hospital discharge). It seems that the recovery of exercise performance was accelerated by PR.

Shareable abstract (@ERSpublications)

In the light of missing randomised controlled trials, some arguments suggest that pulmonary rehabilitation has beneficial effects beyond natural recovery https://bit.ly/3ze2xvw

Cite this article as: Gloeckl R, Leitl D, Jarosch I, et al. Pulmonary rehabilitation in long COVID: more than just natural recovery!? ERJ Open Res 2021; 7: 00454-2021 [DOI: 10.1183/23120541.00454-2021]. 


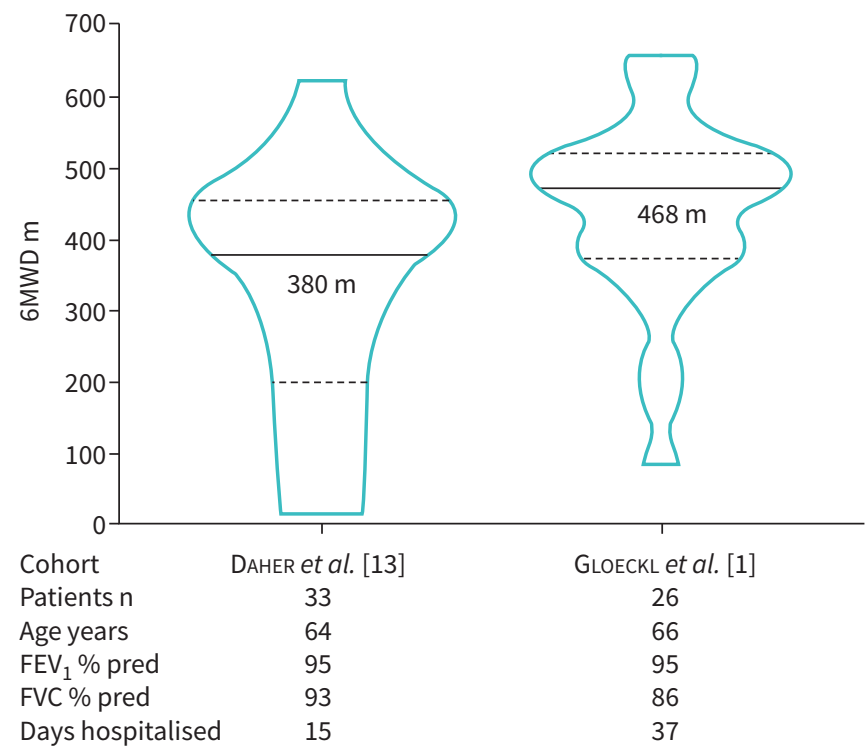

FIGURE 1 Comparison of the 6-min walk distance (6MWD) between two cohorts of coronavirus disease 2019 patients, 6 weeks after hospital discharge. Patients in the cohort from GLOECKL et al. [1] performed comprehensive pulmonary rehabilitation, and data are given from the severe/critical subgroup of patients. Original data from the comparison group was provided courtesy of DAHER et al. [13]. Within the violin plots (showing the distribution shape of the data), the solid horizontal lines, and the values below them, represent the median; dashed lines represent interquartile ranges. Data below the graphs are medians. FEV ${ }_{1}$ : forced expiratory volume in $1 \mathrm{~s}$; FVC: forced vital capacity.

Even clearer is a comparison of our data with another COVID-19 cohort published by DAHER et al. [13], which was comparable in age and lung function. These patients were followed up 6 weeks after hospital discharge. Assessments of COVID-19 patients in our study at the end of PR were also 6 weeks after hospital discharge. This makes the Daher cohort an interesting comparison group, although it remains unknown if and how many patients in this cohort performed PR after hospital discharge. Although their patients had a shorter duration of hospitalisation (37 versus 15 days), the median 6MWD was markedly higher in our severe/critical cohort (following PR) compared to the cohort of DAHER et al. [13] (468 versus $380 \mathrm{~m}$; figure 1).

Another consideration of A. Hussain and co-workers was that our PR service was supposed to differ from standard practice because we offered "only" a 3-week PR programme. We have not mentioned this in the manuscript clearly enough, but a 3-week inpatient multidisciplinary PR is an obligatory procedure in Germany as well as in some other European countries, e.g. in Austria or Switzerland. Several studies have shown that such a comprehensive PR programme is highly beneficial to improve health status and quality of life in patients with chronic respiratory diseases [14-17].

In conclusion, based on all mentioned aspects, we strongly believe that PR has beneficial effects beyond natural recovery. However, we agree with A. Hussain and co-workers that well-planned, randomised controlled trials are necessary, to clarify the relevance of PR in COVID-19 on a higher evidence level. Such trials have already been initiated (e.g. ClinicalTrials.gov identifiers NCT04821934, NCT04365738 and NCT04718506).

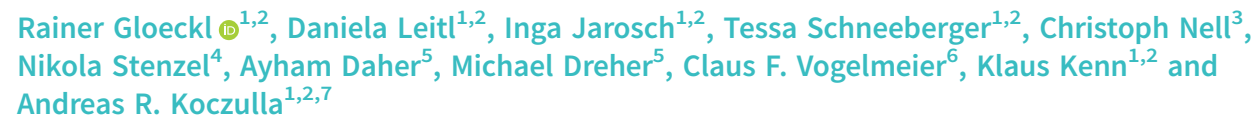

${ }^{1}$ Dept of Pulmonary Rehabilitation, Philipps-University of Marburg, Member of the German Center for Lung Research (DZL), Marburg, Germany. ${ }^{2}$ Institute for Pulmonary Rehabilitation Research, Schoen Klinik Berchtesgadener Land, Schoenau am Koenigssee, Germany. ${ }^{3}$ Dept of Pulmonology, PhilippsUniversity of Marburg, Marburg, Germany. ${ }^{4}$ Psychologische Hochschule Berlin (PHB), Berlin, Germany. 
${ }^{5}$ Dept of Pneumology and Intensive Care Medicine, University Hospital RWTH Aachen, Aachen, Germany. ${ }^{6}$ Dept of Medicine, Pulmonary and Critical Care Medicine, University Medical Centre Giessen and Marburg, Philipps-University of Marburg, Member of the DZL, Marburg, Germany. ${ }^{7}$ Teaching Hospital, Paracelsus Medical University, Salzburg, Austria.

Corresponding author: Rainer Gloeckl (rgloeckl@schoen-klinik.de)

Provenance: Invited article, peer reviewed.

Conflict of interest: None declared.

References

1 Gloeckl R, Leitl D, Jarosch I, et al. Benefits of pulmonary rehabilitation in COVID-19: a prospective observational cohort study. ERJ Open Res 2021; 7: 00108-2021.

2 Greenhalgh T, Knight M, A'Court C, et al. Management of post-acute COVID-19 in primary care. BMJ 2020; 370: m3026.

3 Vaes AW, Goërtz YMJ, Van Herck M, et al. Recovery from COVID-19: a sprint or marathon? 6-month follow-up data from online long COVID-19 support group members. ERJ Open Res 2021; 7: 00141-2021.

4 Sudre CH, Murray B, Varsavsky T, et al. Attributes and predictors of long COVID. Nat Med 2021; 27: 626-631.

5 Negrini F, de Sire A, Andrenelli E, et al. Rehabilitation and COVID-19: update of the rapid living systematic review by Cochrane Rehabilitation Field as of April 30th, 2021. Eur J Phys Rehabil Med 2021; in press [https:// doi.org/10.23736/S1973-9087.21.07125-2].

6 Liu K, Zhang W, Yang Y, et al. Respiratory rehabilitation in elderly patients with COVID-19: a randomized controlled study. Complement Ther Clin Pract 2020; 39: 101166.

7 Liu Y, Yang YQ, Liu Y, et al. Effects of group psychological intervention combined with pulmonary rehabilitation exercises on anxiety and sleep disorders in patients with mild coronavirus disease 2019 (COVID-19) infections in a Fangcang hospital. Psychol Health Med 2021; in press [https://doi.org/10.1080/ 13548506.2021.1916956].

8 AWMF online. S2k-LL SARS-CoV-2, COVID-19 und (Früh-) Rehabilitation [S2k Guideline SARS-CoV-2, COVID-19 and (Early) Rehabilitation]. https://www.awmf.org/uploads/tx_szleitlinien/080-008I_S2k_SARS-CoV-2_COVID-19_ und__Fr\%C3\%BCh___Rehabilitation_2020-11.pdf Date last updated: 1 November 2020. Date last accessed: 24 June 2021.

9 Troosters T, Gosselink R, Decramer M. Six minute walking distance in healthy elderly subjects. Eur Respir J 1999; 14: 270-274.

10 Holland AE, Spruit MA, Troosters T, et al. An official European Respiratory Society/American Thoracic Society technical standard: field walking tests in chronic respiratory disease. Eur Respir J 2014; 44: 1428-1446.

11 Hernandes NA, Wouters EFM, Meijer K, et al. Reproducibility of 6-minute walking test in patients with COPD. Eur Respir J 2011; 38: 261-267.

12 Huang C, Huang L, Wang Y, et al. 6-month consequences of COVID-19 in patients discharged from hospital: a cohort study. Lancet 2021; 397: 220-232.

13 Daher A, Balfanz P, Cornelissen C, et al. Follow up of patients with severe coronavirus disease 2019 (COVID-19): pulmonary and extrapulmonary disease sequelae. Respir Med 2020; 174: 106197.

14 Kenn K, Gloeckl R, Soennichsen A, et al. Predictors of success for pulmonary rehabilitation in patients awaiting lung transplantation. Transplantation 2015; 99: 1072-1077.

15 Huppmann P, Sczepanski B, Boensch M, et al. Effects of inpatient pulmonary rehabilitation in patients with interstitial lung disease. Eur Respir J 2013; 42: 444-453.

16 Schultz K, Wittmann M, Wagner R, et al. In-patient pulmonary rehabilitation to improve asthma control. Dtsch Arztebl Int 2021; 118: 23-30.

17 Jarosch I, Gehlert S, Jacko D, et al. Different training-induced skeletal muscle adaptations in COPD patients with and without alpha-1 antitrypsin deficiency. Respiration 2016; 92: 339-347. 